\title{
Epidemiology and risk factors of Staphylococcus aureus CC398 bone and joint infections
}

Kevin Bouiller ${ }^{1 *}$ (D) Didier Hocquet ${ }^{2,3,4}$, Marlene Sauget ${ }^{2,3,4}$, Xavier Bertrand ${ }^{2,3}$ and Catherine Chirouze ${ }^{1,3}$

\begin{abstract}
Background: A particular ability of the Staphylococcus aureus clonal complex 398 (CC398) to cause bone and joint infections (BJI) remains questionable, since some studies have described high prevalence of MSSA CC398 in prosthetic joint infection (PJI) and diabetic foot ostemolyelitis (DFO). Here, we described the long-term epidemiology of CC398 among S. aureus isolated from BJI and identified risk factors associated with CC398.
\end{abstract}

Methods: We included all bone and joint samples with S. aureus-positive culture in our university hospital between January 2010 and December 2017. Logistic regression was used for univariate and multivariate analysis.

Results: We identified 124 CC398 isolates among the 958 BJ-associated S. aureus. The proportion of CC398 among S. aureus increased steadily from $4 \%$ in 2010 to $26 \%$ in 2017 . Only 4 isolates of CC398 were resistant to methicillin. The distribution of BJI types due to CC398 and non CC398 isolates was similar. In multivariate analysis, age ( $p=$ 0.034, $\mathrm{OR}=3.9)$, McCabe score $(p=0.005, \mathrm{OR}=5)$ and inoculation mechanism $(p=0.020, \mathrm{OR}=3.7)$ were associated with PJI-related CC398. The year of infection ( $p<0.001, \mathrm{OR}=1.6)$, Charlson's score $(p=0.001, \mathrm{OR}=1.5)$ and grade 4 (severe) of the International Working Group of the Diabetic Foot classification $(p<0.001, \mathrm{OR}=8.5)$ were associated with DFO-related CC398.

Conclusion: We highlighted here the emergence and spread of CC398-MSSA in BJI. Patients with comorbidities are at high risk of CC398 MSSA PJI and DFO. The spread of CC398 in the community and hospital settings remains unclear and further epidemiological studies are needed to identify the determinants of its success.

Keywords: Prosthetic joint infection, Bone joint infection, Diabetic foot infection, Human ST398, Methicillin susceptible Staphylococcus aureus, CC398

\section{Background}

Bone and joint infections (BJI) are a heterogeneous disease in their pathophysiology, clinical presentation, and management [1] and Staphylococcus aureus is the most common pathogen in almost all types of BJIs.

Foot ulcers are common in diabetic patients and bone infection is a major causal factor for lower-limb amputation

\footnotetext{
* Correspondence: kbouiller@chu-besancon.fr

${ }^{1}$ Maladies infectieuses et tropicales, Centre Hospitalier Régional Universitaire, 25030 Besancon, France

Full list of author information is available at the end of the article
}

[2]. Prosthetic joint infection (PII) remains a dreaded complication following total joint arthroplasty. PJI is reported in approximately $0.3-1.9 \%$ of all total arthroplasties.

In recent years, infections with $S$. aureus clonal complex 398 (CC398) have emerged worldwide. Whereas MethicillinResistant S. aureus (MRSA) CC398 is associated with livestock and most often responsible of colonization and mild infection in humans and animals [3], Methicillin-Susceptible S. aureus (MSSA) CC398 is a frequent source of infections in humans, and was described frequently in severe infections such as bloodstream infections (BSI) $[4,5]$. Specifically, the

(c) The Author(s). 2020 Open Access This article is licensed under a Creative Commons Attribution 4.0 International License, which permits use, sharing, adaptation, distribution and reproduction in any medium or format, as long as you give appropriate credit to the original author(s) and the source, provide a link to the Creative Commons licence, and indicate if changes were made. The images or other third party material in this article are included in the article's Creative Commons licence, unless indicated otherwise in a credit line to the material. If material is not included in the article's Creative Commons licence and your intended use is not permitted by statutory regulation or exceeds the permitted use, you will need to obtain permission directly from the copyright holder. To view a copy of this licence, visit http://creativecommons.org/licenses/by/4.0/ The Creative Commons Public Domain Dedication waiver (http://creativecommons.org/publicdomain/zero/1.0/) applies to the data made available in this article, unless otherwise stated in a credit line to the data. 
incidence of BSIs due to MSSA CC398 has been increasing since 2007 in France [6].

The frequency of CC398 PJI isolates varied from 1.8 to $14 \%$ [7, 8] whereas $21.7 \%$ of isolates belonged to CC398 in patients with DFO [9]. Since several studies have documented a high prevalence of MSSA CC398 in prosthetic joint infection (PII) and in diabetic foot osteomyelitis (DFO) [6, 7], a particular ability of this clone to cause BJIs remains questionable. However, risk factors for ST398 BJI remain largely unknown. In the study of Valour et al., no difference was observed between patients with MSSA ST398 and non ST398 PJI, excepted for outcome with a less treatment failure in patients with MSSA ST398 infection.

In this study, the prevalence of CC398 among BJI-related $S$. aureus was determined and patients with BJI-related $S$. aureus CC398 were compared to patients with BJI-related S. aureus non-CC398, in a university hospital over 8 years, to identify risk factors associated to infections with CC398. A focus was made on patients with PJI and DFO.

\section{Methods}

Inclusion

All bone and joint bacteriological samples (per-cutaneous joint fluid aspiration, bone or joint surgical sample) with S. aureus positive culture between 1st January 2010 and 31 December 2017 in a French university hospital were retrospectively included.

\section{Definitions}

BJI diagnosis was based on clinical and biological evidence of infection. We extracted the clinical information from the electronic medical records of the patients.

BJIs were classified as follow: (i) orthopaedic implant (i.e. PJI or peripheral or vertebral osteosynthesis) (ii) DFO (iii) BJI related to decubitus ulcer (iv) BJI related to peripheral vascular disease (v) osteomyelitis and (vi) arthritis.

Acute $(\leq 4$ weeks) and chronic infections were defined on the basis of the duration of signs of infection at the prosthesis site to diagnosis.

The Charlson's comorbidity index was calculated as previously described [10]. The McCabe score was also used to evaluate underlying illness severity. Patients were classified as rapidly fatal (<1 year), ultimately fatal (1-4 years) and non-fatal ( $>5$ years) underlying disease [11].

For DFO, we used the IWGDF/IDSA (International Working Group on the Diabetic Foot/ Infectious Diseases Society of America) classification which defined four grades of severity [12]. Grade 1 and 2 infections were only skin and soft tissue infections (SSTI) and were not included in the current study. Grade 3 defined infections in stable patients with local complication such as joint or bone infection. Grade 4 defined infections in patients with systemic toxicity or metabolic instability.

\section{CC398 S. aureus identification}

Only the first isolate from each patient was included. $S$. aureus isolates retrieved from bone and joint cultures were stored at the Centre de Ressources Biologiques Filière Microbiologique, Besançon (CRB-FMB, Biobanque BB0033-00090). MALDI-TOF MS Microflex LT identified all the isolates as $S$. aureus with a $\log$ score value $\geq 2$ according to the manufacturer's recommendations (Bruker Daltonik $\mathrm{GmbH}$, Bremen, Germany). Cefoxitin susceptibility was determined using the disk diffusion method according to EUCAST recommendations (www.eucast.org). We used a CC398-lineage specific MALDI-TOF MS method to screen all $S$. aureus isolates, as described previously $[13,14]$ and CC398 status was confirmed by a CC398-lineage specific PCR [15].

\section{Statistical analysis}

All variables were examined by univariate analysis using the chi-square or Fisher's exact test, as appropriate. Continuous variables were analyzed by Student's t-test. All statistical tests were two tailed and $p<0.05$ was considered statistically significant. Multivariate analysis was performed by logistic regression. A backward stepwise selection with an entry and stay level of $p=0.2$ was used to build the final multivariate logistic regression model. Statistical analyses were computed by SPSS 22.0 (IBM, Armonk, NY, USA).

\section{Results}

Over the eight-year survey, 1080 non-duplicate S.aureus BJI were isolated from inpatients of our hospital. Among these, 958 (88.7\%) were available for further analysis. Within this S. aureus BJI collection, 124 (12.9\%) isolates belonged to CC398. Only 4 isolates were resistant to methicillin (one isolate in 2013 and 2015, and 2 isolates in 2017). The proportion of CC398 among S. aureus isolates increased steadily from $4 \%$ in 2010 to $26 \%$ in 2017 $(p<0.001)$ (Fig. 1).

Distribution of the 124 BJI-related S. aureus CC398 was showed in Table 1. The distribution of the types of BJIs due to isolates of S. aureus CC398 and non-CC398 was similar (Table 1).

A total of 144 patients had a S. aureus PJI (12.9\%), with 23 due to CC398 isolates (Table 2). Patients with $S$. aureus CC398 PJI were younger $(p=0.041)$, had more severe illness (McCabe score) $(p=0.016)$, were more likely to have early PJI ( $<3$ months after primary arthroplasty) $(p=$ 0.023 ) and had an inoculation mechanism more frequent $(p=0.032)$ than patients with $S$. aureus non-CC398 PJI. In multivariate analysis, age $(p=0.034$, OR $=3.9(1.3-12.0))$, McCabe score $(p=0.005, \mathrm{OR}=5(1.6-15.3))$ and inoculation mechanism $(p=0.020$, OR $=3.7(1.2-11.3))$ were associated with PJI due to $S$. aureus CC398. 


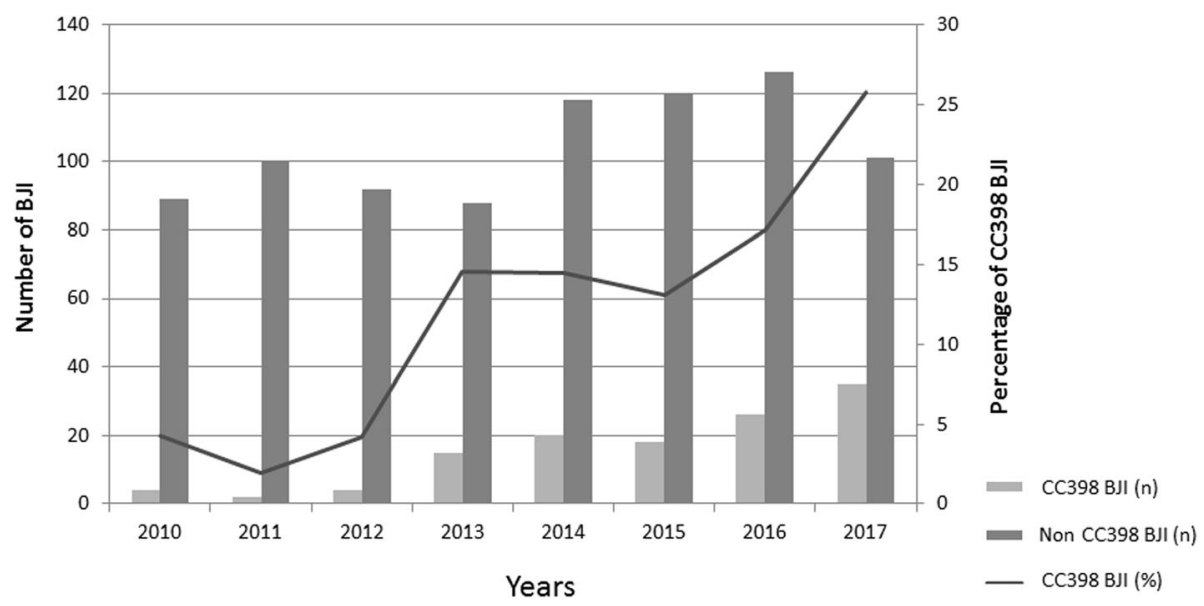

Fig. 1 Proportion of Staphylococcus aureus (SA) belonging to the complex clonal $398(n=124)$ among the SA retrieved in Bone joint and infection ( $n=958$ ) between 2010 and 2017 in the University Hospital of Besancon, France

Of the 244 patients with DFO (25.5\%), 37 were infected with CC398 isolates (Table 3). A higher Charlson's comorbidity score $(p<0.001)$, and grade 4 of the IWGDF/IDSA classification were more frequent in patients with DFO due to $S$. aureus CC398 than in patients with non-CC398 isolates $(p<0.001)$. Multivariate analysis associated the year of infection $(p<0.001, \mathrm{OR}=1.6(1.3-2))$, the Charlson's score $(p=0.001, \mathrm{OR}=1.5(1.2-2))$ and grade 4 of the IWGDF/ IDSA classification $(p<0.001, \mathrm{OR}=8.5(3.5-20.7))$ with DFO due to $S$. aureus CC398.

\section{Discussion}

In our hospital, the number of BJIs due to $S$. aureus was stable between 2010 and 2013 and then increased in 2014 to stabilize until 2017. However, the prevalence of CC398 among S. aureus responsible for BJIs increased steadily from $4 \%$ in 2010 to $26 \%$ in 2017 . Our results describe the increasing trend of MSSA CC398 in BJIs over a 8-year period, echoing what we observed in bloodstream infections in our hospital [4].

The spread of this clone has also been reported in BJIs in other French hospitals with a prevalence varying from 1.8 to $21.7 \%[7-9,16,17]$. These differences were explained by different study periods and characteristics of some types of BJI. Indeed, the frequency of CC398 PJI isolates varied from $1.8 \%$ from $14 \%$ [ 7,8$]$, whereas $21.7 \%$ of isolates belonged to CC398 in patients with DFO [9]. Interestingly, Senneville et al. showed that $S$. aureus CC398 isolates were significantly more frequent in osteomyelitis than in SSTI for patients with diabetic foot infection and suggested the possible tropism for bone of this clone [9].

This clonal group has been also described in BJIs elsewhere over the world. Uhlemann et al. identified 3 out of 64 (4.7\%) CC398 positive samples with osteomyelitis in the United States [18].
Recently, ST398 was found to be the most prevalent clone in BSI-related MSSA in China (14.1\%, 32/227), and the prevalence of MSSA ST398 increased from 2013 to 2019 (5.5-18.4\%). However, characteristics of BSI including the presence of BJI were not described [5].

Although this clone spread globally, BJI infections with MSSA CC398 had specially been reported in France. It can be explained by the limited surveillance of MSSA in other countries and because most previous international staphylococcal clonal distribution studies have mainly focused on MRSA.

As expected, only 4 isolates were resistant to methicillin. It has been shown that MRSA ST398 and MSSA ST398 belonged to distinct lineages [19]. MRSA ST398 lineage was mainly associated with livestock. It has become a worldwide threat within the past decade and was most often responsible for mild infections, such as SSTI. Few studies had described MRSA CC398 in BJI [20-22]. In contrast, MSSA CC398 was a frequent source of $S$. aureus human infections, and was described frequently in severe infections such as BSI $[5,6,18,23]$. No contact with livestock was found for the 4 patients with MRSA CC398 isolates. We assume that these 4 isolates were MSSA CC398 from human origin that acquired mecA resistance gene but further studies are needed to clarify this point.

The low resistance profile of this clone was confirmed from different studies, who found no particular resistance gene (except for an isolated resistance to macrolides related to the ermT gene). Regarding the virulence profile, none of the major, most well-known staphylococcal virulence genes were described in CC398 isolates, except in China, where PVL toxin was found in $80 \%$ of CC398 MSSA [5]. However, almost all isolates harboured mobile genetic element belonging to the immune evasion cluster (chp and scn genes) and the $\phi 3$-prophage specific from human lineage and lost in the animal-adapted MRSA CC398 [8, 24, 25]. 
Table 1 Characteristics of patients with CC398 Bone and joint infections

\begin{tabular}{|c|c|c|c|}
\hline & CC $398(n=124)$ & Non CC $398(n=834)$ & Univariate $P$ value \\
\hline Age (years), mean $\pm S D$ & $62.2( \pm 18.6)$ & $64.3( \pm 18.7)$ & 0.24 \\
\hline Male sex, n (\%) & $91(73)$ & $573(69)$ & 0.34 \\
\hline \multicolumn{4}{|l|}{ Mc Cabe Score } \\
\hline Non fatal, n (\%) & $110(88.7)$ & $761(91.2)$ & \multirow[t]{3}{*}{0.19} \\
\hline Ultimately fatal, n (\%) & $13(10.5)$ & $63(7.6)$ & \\
\hline Rapidly fatal, n (\%) & $1(0.8)$ & $10(1.2)$ & \\
\hline MSSA, n (\%) & $120(97)$ & $701(84)$ & $<0.001$ \\
\hline Charlson's comorbidity score, mean \pm SD & $2.46( \pm 2.22)$ & $2.08( \pm 1.94)$ & 0.073 \\
\hline \multicolumn{4}{|l|}{ Year of infection } \\
\hline 2010 & $4(3.2)$ & $89(11)$ & \multirow[t]{8}{*}{$<0.001$} \\
\hline 2011 & $2(1.6)$ & $100(12)$ & \\
\hline 2012 & $4(3.2)$ & $92(11)$ & \\
\hline 2013 & $15(12)$ & $88(11)$ & \\
\hline 2014 & $20(16)$ & $118(14)$ & \\
\hline 2015 & $18(15)$ & $120(14)$ & \\
\hline 2016 & $26(21)$ & $126(15)$ & \\
\hline 2017 & $35(28)$ & $101(12)$ & \\
\hline Recent hospitalisation (< 1 yr), n (\%) & $62(50)$ & $474(57)$ & 0.18 \\
\hline Surgery (< 1 yr), n (\%) & $68(55)$ & $428(51)$ & 0.53 \\
\hline \multicolumn{4}{|l|}{ BJ Type } \\
\hline Arthritis, n (\%) & $8(6.5)$ & $47(5.6)$ & 0.87 \\
\hline Osteomyelitis, n (\%) & $15(12,1)$ & $119(14.3)$ & 0.52 \\
\hline Vertebral osteomyelitis, n (\%) & 0 & $6(0.7)$ & 1 \\
\hline Diabetic foot osteomyelitis, n (\%) & $37(29.8)$ & $207(24.8)$ & 0.28 \\
\hline BJI related to decubitus ulcer, $\mathrm{n}(\%)$ & $4(3.2)$ & $40(4.8)$ & 0.58 \\
\hline BII related to peripheral vascular disease & $7(5.7)$ & $101(12.1)$ & 0.05 \\
\hline Orthopaedic device infection, n (\%) & $53(42.7)$ & $314(37.7)$ & 0.31 \\
\hline including PJI, n (\%) & $23(18.6)$ & $121(14.5)$ & 0.3 \\
\hline including osteosynthesis infection, n (\%) & $29(23.4)$ & $184(22.1)$ & 0.83 \\
\hline including vertebral ODI, n (\%) & $1(0.8)$ & $9(1.1)$ & 1 \\
\hline \multicolumn{4}{|l|}{ BJl characteristics } \\
\hline Evolution delay (months) & $28( \pm 62.9)$ & $37.6( \pm 101.7)$ & 0.32 \\
\hline Chronic BJI (i.e. evolution delay > 4 weeks), n (\%) & $49(75)$ & $270(68)$ & 0.29 \\
\hline Bacteremiae, n (\%) & $20(16)$ & $87(10)$ & 0.084 \\
\hline \multicolumn{4}{|l|}{ BJI mechanisms } \\
\hline Haematogenous, n (\%) & $4(3.2)$ & $49(5.9)$ & 0.32 \\
\hline Inoculation, n (\%) & $62(50)$ & $369(44)$ & 0.27 \\
\hline Contiguity, n (\%) & $58(47)$ & $416(50)$ & 0.58 \\
\hline
\end{tabular}

MSSA CC398 isolates could be more virulent than non-CC398, as suggested by the association of grade 4 of the IWGDF/IDSA classification in patients with $S$. aureus CC398 DFO. However, this may also reflect underlying host comorbidities and immunosuppression. This hypothesis was confirmed in our study with a high
Charlson's score index in patients with SA CC398 DFO, and a high McCabe score in patients with SA CC398 PJI. Similarly, we reported in another study that the 30day all-cause mortality and McCabe score were higher for patients with MSSA CC398 BSI than those with nonCC398 BSI [23]. Moreover, in a study comparing eight 
Table 2 Characteristics of patients with CC398 PJ

\begin{tabular}{|c|c|c|c|c|c|}
\hline & \multirow{2}{*}{$\begin{array}{l}\text { CC } 398 \text { PJI } \\
(n=23)\end{array}$} & \multirow{2}{*}{$\begin{array}{l}\text { Non CC } \\
398 \text { PJI } \\
(n=121)\end{array}$} & \multirow{2}{*}{$\begin{array}{l}\text { Univariate } \\
P \text { value }\end{array}$} & \multicolumn{2}{|c|}{ Multivariate } \\
\hline & & & & $P$ value & OR (IC 95\%) \\
\hline Age (years), mean $\pm S D$ & $67.7( \pm 12.3)$ & $73.6( \pm 12.3)$ & 0.041 & 0.034 & $0.9(0.9-0.99)$ \\
\hline Male sex, n (\%) & $11(48)$ & $65(54)$ & 0.77 & & \\
\hline \multicolumn{6}{|l|}{ Mc Cabe Score } \\
\hline Non fatal, n (\%) & $18(78)$ & $114(94.2)$ & 0.016 & 0.005 & $5.1(1.6-15.3)$ \\
\hline Ultimately fatal, n (\%) & $5(22)$ & $6(5)$ & & & \\
\hline Rapidly fatal, n (\%) & $0(0)$ & $1(0.8)$ & & & \\
\hline MSSA, n (\%) & $23(100)$ & $108(89)$ & 0.13 & & \\
\hline Charlson's comorbidity score, mean \pm SD & $2.04( \pm 1.89)$ & $1.56( \pm 1.61)$ & 0.27 & & \\
\hline \multicolumn{6}{|l|}{ Year of infection } \\
\hline 2010 & $2(8.7)$ & $14(11)$ & 0.1 & NT & \\
\hline 2011 & $2(8.7)$ & $9(7.3)$ & & & \\
\hline 2012 & $2(8.7)$ & $18(15)$ & & & \\
\hline 2013 & $4(17)$ & $13(11)$ & & & \\
\hline 2014 & $1(4.3)$ & $17(14)$ & & & \\
\hline 2015 & $2(8.7)$ & $16(13)$ & & & \\
\hline 2016 & $2(8.7)$ & $24(20)$ & & & \\
\hline 2017 & $8(35)$ & $12(9.8)$ & & & \\
\hline Recent hospitalisation (< 1 yr), n (\%) & $13(57)$ & $74(60)$ & 0.92 & & \\
\hline \multicolumn{6}{|l|}{ BJl Type } \\
\hline Shoulder, n (\%) & $1(4.3)$ & $5(4.1)$ & 1 & & \\
\hline Hip, n (\%) & $16(70)$ & $75(62)$ & 0.65 & & \\
\hline Knee, n (\%) & $6(26)$ & $40(33)$ & 0.68 & & \\
\hline Ankle, n (\%) & 0 & $1(0.8)$ & 1 & & \\
\hline Chronic BJI (i.e. evolution delay > 4 weeks) & $5(28)$ & $51(42)$ & 0.37 & & \\
\hline \multicolumn{6}{|c|}{ Occurrence of infection after primary arthroplasty } \\
\hline Delay (months) & $23( \pm 49.3)$ & $44.4( \pm 59.2)$ & 0.023 & NT & \\
\hline$<3$ months, $\mathrm{n}(\%)$ & $14(61)$ & $44(36)$ & 0.049 & NT & \\
\hline 3-12 months, n (\%) & $3(13)$ & $17(14)$ & 1 & & \\
\hline 12 months, $n$ (\%) & $7(30)$ & $60(50)$ & 0.14 & & \\
\hline Bacteremia, n (\%) & $8(35)$ & $32(26)$ & 0.57 & & \\
\hline \multicolumn{6}{|l|}{ BJI mechanisms } \\
\hline haematogenous, n (\%) & $3(13)$ & $37(31)$ & 0.14 & & \\
\hline inoculation, n (\%) & $20(87)$ & $74(61)$ & 0.032 & 0.020 & $3.7(1.2-11.3)$ \\
\hline Contiguity, n (\%) & 0 & $10(8.3)$ & 0.36 & & \\
\hline \multicolumn{6}{|l|}{ Clinical features } \\
\hline Fever, n (\%) & $10(43)$ & $48(40)$ & 0.66 & & \\
\hline Sinus tract, $\mathrm{n}(\%)$ & $7(30)$ & $48(40)$ & 1 & & \\
\hline Abscess, n (\%) & $3(13)$ & $12(9.9)$ & 0.4 & & \\
\hline \multicolumn{6}{|l|}{ Biological features } \\
\hline Maximum CRP value (mg/L) & $163( \pm 69.1)$ & $171( \pm 120)$ & 0.9 & & \\
\hline
\end{tabular}

MSSA Methicillin susceptible Staphylococcus aureus, BJ Bone and joint infection, NT Not included in the final model 
Table 3 Characteristics of patients with CC398 diabetic foot infection

\begin{tabular}{|c|c|c|c|c|c|}
\hline & \multirow{2}{*}{$\begin{array}{l}\text { CC } 398 \text { DFI } \\
(n=37)\end{array}$} & \multirow{2}{*}{$\begin{array}{l}\text { Non CC } \\
398 \text { DFI } \\
(n=207)\end{array}$} & \multirow{2}{*}{$\begin{array}{l}\text { Univariate } \\
P \text { value }\end{array}$} & \multicolumn{2}{|c|}{ Multivariate } \\
\hline & & & & $P$ value & OR (IC 95\%) \\
\hline Age (years), mean $\pm S D$ & $69.3( \pm 12.5)$ & $70.3( \pm 11.3)$ & 0.65 & & \\
\hline Male sex, n (\%) & $28(76)$ & $166(80)$ & 0.68 & & \\
\hline \multicolumn{6}{|l|}{ Mc Cabe Score } \\
\hline Non fatal, n (\%) & $34(91.9)$ & $192(92.8)$ & 0.81 & & \\
\hline Ultimately fatal, n (\%) & $3(8.1)$ & $14(6.7)$ & & & \\
\hline Rapidly fatal, n (\%) & $0(0)$ & $1(0.5)$ & & & \\
\hline MSSA, n (\%) & $36(97.3)$ & $171(83)$ & 0.013 & NT & \\
\hline Charlson's comorbidity score, mean \pm SD & $4.51( \pm 1.71)$ & $3.48( \pm 1.48)$ & $<0.001$ & 0.001 & $1.5(1.2-2)$ \\
\hline Year of infection & & & $<0.001$ & $<0.001$ & $1.6(1.3-2)$ \\
\hline 2010 & $0(0)$ & $29(14)$ & & & \\
\hline 2011 & $0(0)$ & $38(18)$ & & & \\
\hline 2012 & $2(5.4)$ & $23(11)$ & & & \\
\hline 2013 & $4(11)$ & $25(12)$ & & & \\
\hline 2014 & $6(16)$ & $24(12)$ & & & \\
\hline 2015 & $4(11)$ & $24(12)$ & & & \\
\hline 2016 & $10(27)$ & $22(11)$ & & & \\
\hline 2017 & $11(30)$ & $22(11)$ & & & \\
\hline Recent hospitalisation $(<1 \mathrm{yr}), \mathrm{n}(\%)$ & $21(57)$ & $102(49)$ & 0.51 & & \\
\hline Bacteremia, n (\%) & $3(8.1)$ & $12(5.8)$ & 0.71 & & \\
\hline \multicolumn{6}{|l|}{ IWGDF-IDSA grade } \\
\hline 3 (moderate), n (\%) & $14(38)$ & $174(84)$ & $<0.001$ & $<0.001$ & $8.5(3.5-20.7)$ \\
\hline 4 (severe), n (\%) & $23(62)$ & $33(16)$ & & & \\
\hline
\end{tabular}

MSSA Methicillin susceptible Staphylococcus aureus, IDSA Infectious Diseases Society of America, IWGDF International Working Group of the Diabetic Foot, NT Not included in the final model

PJIs with MSSA CC398 to 67 PJIs with other clones of MSSA, no demographic and clinical differences were observed between these 2 groups. Conversely, MSSA CC398 BJIs were significantly associated with a lower biological inflammatory syndrome and lower treatment failure rates $(0 \%$ vs. $37.3 \%)$. However a very small number of patients were included and a large number of statistical tests have been performed, which make these results difficult to interpret [8].

The mechanism of acquisition of this clone may be discussed. CC398 MSSA PJI was associated with inoculation mechanisms (mostly post-surgical). Moreover, in our study, more than $90 \%$ of patients with DFO were hospitalized in the same medical unit (endocrinology ward) and similarly, patients with PJI or device-associated infection were hospitalized in the same surgical ward. However, other patients $(n=40 / 124)$ were hospitalized in various wards of our hospital (medical wards $n=10$, other surgical wards $n=25$, intensive care units $n=5$ ), with no overlapping hospital stays and were taken care by different surgeons in different surgical rooms. Because of the many cases in the same ward and the association with inoculation mechanism after surgery, we cannot rule out hospital cross-transmission. However, the diffusion of this clone in different hospitals in France and in other countries may suggest that this clone spread in both the community and hospital settings. Interestingly, heterogeneity in geographical distribution was observed in France with a prevalence of 3.1 to $23.5 \%$ according to the hospital [8]. This heterogeneity could be explained by a greater endemic diffusion in some areas, possibly related to specific routes of transmission.

Our study has some limitations. Firstly, the retrospective collection of data may have introduced information bias. Secondly, we did not determine the population structure of non-CC398, and the control group very probably consisted of genetically diverse $S$. aureus.

\section{Conclusion}

Our local data confirm the emergence and the dissemination of MSSA CC398 in BJI, suggesting a well-adapted fitness of this clone to humans and bone.

Patients with comorbidities are at high risk of MSSA CC398 PJIs and DFOs. The mode of diffusion of this clone in community and hospital remain unclear and further epidemiological studies, based on genome exploration, are needed. 


\section{Abbreviations}

BJI: Bone and joint infection; BSI: Bloodstream infection; CC398: Clona complex 398; DFO: Diabetic foot ostemolyelitis; MRSA: Methicillin-Resistant S. aureus; MSSA: Methicillin-Susceptible S. aureus; PJI: Prosthetic joint infection; SSTI: Skin and soft tissue infections

\section{Acknowledgements}

None.

\section{Authors' contributions}

X.B., K. B, D. H and C. C conceived the study. K.B. collected and analyzed the data. K.B. wrote the manuscript with support from D.H., X. B and C.C. M.S. performed bacteriological analysis. All authors provided critical feedback and helped shape the research, analysis and manuscript. The author(s) read and approved the final manuscript.

\section{Funding}

None.

\section{Availability of data and materials}

The datasets used and/or analysed during the current study are available from the corresponding author on reasonable request.

\section{Ethics approval and consent to participate}

According to French legislation in this period, and because no intervention was performed on patients, no written informed consent was given by the patients (Law No. 2012-300 of 5 March 2012: https:/ www.legifrance.gouv.fr/affichTexte.do?cidTexte=JORFTEXT0000254415 $87 \&$ categorieLien=id). Our study protocol followed the ethical guidelines of the declaration of Helsinki and was approved by our institutional review board (Comité de Protection des Personnes - Est (GIRCI Grand Est) $N^{\circ}$ 2020-A02771-54.

\section{Consent for publication}

Not applicable.

\section{Competing interests}

None.

\section{Author details}

${ }^{1}$ Maladies infectieuses et tropicales, Centre Hospitalier Régional Universitaire, 25030 Besancon, France. ${ }^{2}$ Hygiène hospitalière - Centre Hospitalier Régional Universitaire, 25030 Besançon, France. ${ }^{3}$ UMR-CNRS 6249

Chrono-environnement, Université Bourgogne Franche-Comté, 25000

Besançon, France. ${ }^{4}$ Centre de Ressources Biologiques Filière Microbiologique de Besançon, Centre Hospitalier Régional Universitaire, Besançon, France.

\section{Received: 5 February 2020 Accepted: 17 May 2020}

Published online: 29 May 2020

\section{References}

1. Lew DP, Waldvogel FA. Osteomyelitis. Lancet Lond Engl. 2004;364:369-79.

2. Lavery LA, Armstrong DG, Wunderlich RP, Mohler MJ, Wendel CS, Lipsky BA. Risk factors for foot infections in individuals with diabetes. Diabetes Care. 2006;29:1288-93.

3. Becker K, Ballhausen B, Kahl BC, Köck R. The clinical impact of livestockassociated methicillin-resistant Staphylococcus aureus of the clonal complex 398 for humans. Vet Microbiol. 2017:200:33-8.

4. Sauget M, Bouiller K, Richard M, Chagrot J, Cholley P, Hocquet D, et al. Increasing incidence of bloodstream infections due to Staphylococcus aureus clonal complex 398 in a French hospital between 2010 and 2017. Eur J Clin Microbiol Infect Dis Off Publ Eur Soc Clin Microbiol. 2019;38(11):2127.

5. Li X, Huang T, Xu K, Li C, Li Y. Molecular characteristics and virulence gene profiles of Staphylococcus aureus isolates in Hainan, China. BMC Infect Dis. 2019;19:873

6. Valentin-Domelier A-S, Girard M, Bertrand X, Violette J, François P, Donnio P$Y$, et al. Methicillin-susceptible ST398 Staphylococcus aureus responsible for bloodstream infections: an emerging human-adapted subclone? PLoS One. 2011;6:e28369.
7. Aubin GG, Lepelletier D, Reynaud A, Lavigne J-P, Corvec S. Methicillinsusceptible Staphylococcus aureus CC398: first description in prosthetic joint infection and genetic background comparison with nasal carriage isolates. J Infect Chemother. 2014;20:394-6.

8. Valour F, Tasse J, Trouillet-Assant S, Rasigade J-P, Lamy B, Chanard E, et al. Methicillin-susceptible Staphylococcus aureus clonal complex 398: high prevalence and geographical heterogeneity in bone and joint infection and nasal carriage. Clin Microbiol Infect. 2014;20:0772-5.

9. Senneville $E$, Brière $M$, Neut $C$, Messad N, Lina G, Richard J-L, et al. First report of the predominance of clonal complex 398 Staphylococcus aureus strains in osteomyelitis complicating diabetic foot ulcers: a national French study. Clin Microbiol Infect. 2014;20: O274 http://onlinelibrary.wiley.com/doi/10.1111/1469-0691.12375/full. Accessed 4 Oct 2017

10. Charlson M, Szatrowski TP, Peterson J, Gold J. Validation of a combined comorbidity index. J Clin Epidemiol. 1994;47:1245-51.

11. MCCABE WR. Gram-negative bacteremia: I. etiology and ecology. Arch Intern Med. 1962;110:847.

12. Guidelines. IWGDF Guidelines. https://iwgdfguidelines.org/guidelines/ guidelines/. Accessed 7 Oct 2019.

13. Sauget M, van der Mee-Marquet N, Bertrand X, Hocquet D. Matrixassisted laser desorption ionization-time of flight mass spectrometry can detect Staphylococcus aureus clonal complex 398. J Microbiol Methods. 2016;127:20-3.

14. Sauget M, Cholley P, Vannier A, Thouverez M, Nicolas-Chanoine $M-H$, Hocquet $D$, et al. Trends of extended-spectrum $\beta$-lactamase-producing Escherichia coli sequence type 131 and its $\mathrm{H} 30$ subclone in a French hospital over a 15-year period. Int J Antimicrob Agents. 2016;48:744-7.

15. Stegger M, Lindsay JA, Moodley A, Skov R, Broens EM, Guardabassi L. Rapid PCR detection of Staphylococcus aureus clonal complex 398 by targeting the restriction-modification system carrying sau1-hsdS1. J Clin Microbiol. 2011;49:732-4.

16. Bonnet I, Millon B, Meugnier $H$, Vandenesch $F$, Maurin M, Pavese $P$, et al. High prevalence of spa type $\mathrm{t} 571$ among methicillin-susceptible Staphylococcus aureus from bacteremic patients in a French University hospital. PLoS One. 2018;13:10. https://doi.org/10.1371/journal.pone. 0204977.

17. Chroboczek T, Boisset S, Rasigade J-P, Tristan A, Bes M, Meugnier $H$, et al. Clonal complex 398 methicillin susceptible Staphylococcus aureus: a frequent unspecialized human pathogen with specific phenotypic and genotypic characteristics. PLoS One. 2013;8:e68462.

18. Uhlemann A-C, Hafer C, Miko BA, Sowash MG, Sullivan SB, Shu Q, et al. Emergence of sequence type 398 as a community- and healthcareassociated methicillin-susceptible Staphylococcus aureus in northern Manhattan. Clin Infect Dis. 2013;57:700-3.

19. Smith TC, Wardyn SE. Human infections with Staphylococcus aureus CC398. Curr Environ Health Rep. 2015;2:41-51.

20. van Rijen MML, Van Keulen PH, Kluytmans JA. Increase in a Dutch hospital of methicillin-resistant Staphylococcus aureus related to animal farming. Clin Infect Dis Off Publ Infect Dis Soc Am. 2008;46:261-3.

21. Van Hoecke $H$, Piette A, De Leenheer E, Lagasse N, Struelens M, Verschraegen $\mathrm{G}$, et al. Destructive otomastoiditis by MRSA from porcine origin. Laryngoscope. 2009;119:137-40.

22. Sun $L$, Chen $Y$, Wang D, Wang $H$, Wu D, Shi $K$, et al. Surgical site infections caused by highly virulent methicillin-resistant Staphylococcus aureus sequence type 398. China Emerg Infect Dis. 2019;25:157-60.

23. Bouiller K, Gbaguidi-Haore H, Hocquet D, Cholley P, Bertrand X, Chirouze C. Clonal complex 398 methicillin-susceptible Staphylococcus aureus bloodstream infections are associated with high mortality. Clin Microbiol Infect. 2016;22:451-5.

24. Price LB, Stegger M, Hasman H, Aziz M, Larsen J, Andersen PS, et al. Staphylococcus aureus CC398: host adaptation and emergence of methicillin resistance in livestock. mBio. 2012;3:1.

25. Diene SM, Corvaglia AR, François $P$, van der Mee-Marquet N. Regional infection control Group of the Centre Region. Prophages and adaptation of Staphylococcus aureus ST398 to the human clinic. BMC Genomics. 2017;18:133.

\section{Publisher's Note}

Springer Nature remains neutral with regard to jurisdictional claims in published maps and institutional affiliations. 